\title{
Improving fatigue performance of surface-damaged Ti-6Al-4V alloy via ultrasonic surface rolling process
}

\author{
Ni Ao, Daoxin Liu*, Xiaohua Zhang \\ College of Aeronautics, Northwestern Polytechnical University, Xi'an 710072, China \\ * Corresponding author: Tel. : +86 2988491479 ; \\ E-mail: liudaox@nwpu.edu.cn (Daoxin Liu)
}

\begin{abstract}
Surface damages caused by markings, like laser marking and mechanical marking are detrimental to the fatigue performance of the components. To improve the fatigue performance of laser marked or mechanical marked Ti-6Al-4V alloy, ultrasonic surface rolling process (USRP) was performed on Ti-6Al-4V alloy prior to marking. The results showed that laser marking induced a porous and loose oxidized layer which severely decreased the fatigue performance of the substrate. And the mechanical marking slightly decreased the fatigue performance of the substrate due to the local stress concentration at the bottom of the pits. After USRP pretreatment, the detrimental effect of laser marking on the fatigue performance of the substrate was eliminated while the fatigue performance of mechanical marking sample was significantly improved by a factor of 3 . That was because the USRP-induced residual compressive stress suppressed the crack early propagation.
\end{abstract}

Key words: Titanium alloy; Fatigue; Ultrasonic surface rolling process; Surface damage; Residual compressive stress; Laser marking, Mechanical marking

\section{Introduction}

Data matrix (DM) codes have been widely used in the advertising, internet, electronic ticket business and industry due to its small area, large amounts of information, high reliability, high error-correcting ability and high security [1-3]. There are many methods, like ink-marking, electro-chemical methods, mechanical marking and laser marking to mark DM codes to the products [1]. Among them, laser marking produces permanent, high contrast codes rapidly with relatively low cost [3]. However, the laser marking causes a large surface damage on the substrate which is not conducive to its fatigue properties. Mechanical marking could also produce a permanent code with relatively small surface damage on the substrate. Titanium alloys are attractive due to their high specific strength, excellent corrosion resistance and biocompatibility [4]. However, titanium alloys are sensitive to surface notch effect. Thus, it is necessary to investigate the fatigue performance of surface-damaged titanium alloy.

Ultrasonic surface rolling process (USRP), an emerging surface mechanical treatment, could induce a deep-distributed compressive residual stress layer and an excellent surface integrity $[5,6]$. It is reported that USRP could significantly improve the 
fatigue performance of materials $[5,6]$. Therefore, it is proposed that USRP treatment could be performed prior to laser marking or mechanical marking to improve the fatigue performance.

In the present study, Ti-6Al-4V alloy, the most widely used titanium alloy all over the world, is chosen as the substrate. The fatigue performance of the base material (BM), laser marked sample (LM), USRP-laser marked sample (USRP-LM), mechanical marked sample (MM) and USRP-mechanical marked sample (USRP-MM) is investigated.

\section{Experimental details}

\subsection{Experimental material}

The material used in this study was received as a bar of Ti-6Al-4V alloy supplied by Baotai Co. Ltd. in China. The nominal chemical composition, heat treatment condition and the mechanical properties of the Ti-6Al-4V alloy had been elaborated in Ref [7]. The dog-bone shaped fatigue specimens (the working section diameter is $\Phi 6 \mathrm{~mm}$ ) were machined from a $\Phi 15 \mathrm{~mm}$ bars for conducting the fatigue tests.

\subsection{Marking methods and USRP parameters}

A YLP-D10 optical fiber laser marking machine and a TMP6100 mechanical marking machine were used in the experiment. The marking speed of $400 \mathrm{~mm} / \mathrm{s}$, laser power of $68 \mathrm{~W}$, scanning pitch of $0.02 \mathrm{~mm}$ and module indent of $0.056 \mathrm{~mm}$ were used in laser marking. In mechanical marking, the marking speed of 50\%, the pressure of $3 \mathrm{MPa}$ and the distance of $6 \mathrm{~mm}$ were used.

The specimens were subjected to USRP under a static force of $900 \mathrm{~N}$ using a rotation speed of $200 \mathrm{rev} / \mathrm{min}$ and the feeding rate is $0.10 \mathrm{~mm} / \mathrm{rev}$. A scrollable rolling WC/Co ball with a hardness of $80 \mathrm{HRC}$, surface roughness of Ra $0.1 \mu \mathrm{m}$ and radius of $14 \mathrm{~mm}$ equipped with an ultrasonic apparatus was used for process. The material was subjected to an ultrasonic vibration frequency and ultrasonic vibration amplitude of $20 \mathrm{kHz}$ and $20 \mu \mathrm{m}$, respectively.

\subsection{Material characterization}

The surface morphologies and fracture morphologies of all the samples were observed using scanning electron microscopy (SEM) (JEOL JSM-6390). The element content was detected by energy dispersive spectrum (EDS) instrument attached on the SEM device.

\subsection{Fatigue test}

To evaluate the effect of different surface damages on the fatigue life of Ti-6Al-4V alloy, rotating bending fatigue tests were performed at a PQ-6 type rotating bending fatigue tester at room temperature. A maximum alternating stress of $700 \mathrm{MPa}$ was chosen. A minimum of two samples were tested for base material (BM), laser marked sample (LM), USRP-laser marked sample (USRP-LM), mechanical marked sample (MM) and USRP-mechanical marked sample (USRP-MM).

\section{Results and discussion}




\subsection{Morphologies of DM codes}

Fig. 1 shows the morphologies of laser marked DM code and mechanical marked DM code on the titanium alloy surface. After laser treatment, a porous and loose layer with a depth of 40 60 $\mu \mathrm{m}$ was formed on the titanium alloy surface. And the treated area had been oxidized via the EDS analysis. The porous and loose layer were easy to crack under the fatigue loading [8]. Many literatures showed that the porous and loose surface layer was harmful to its mechanical properties [9]. After mechanical marking, many pits with a depth of 30 35 $\mu \mathrm{m}$ were formed on the titanium alloy. And the bottom of the pits was blunt.
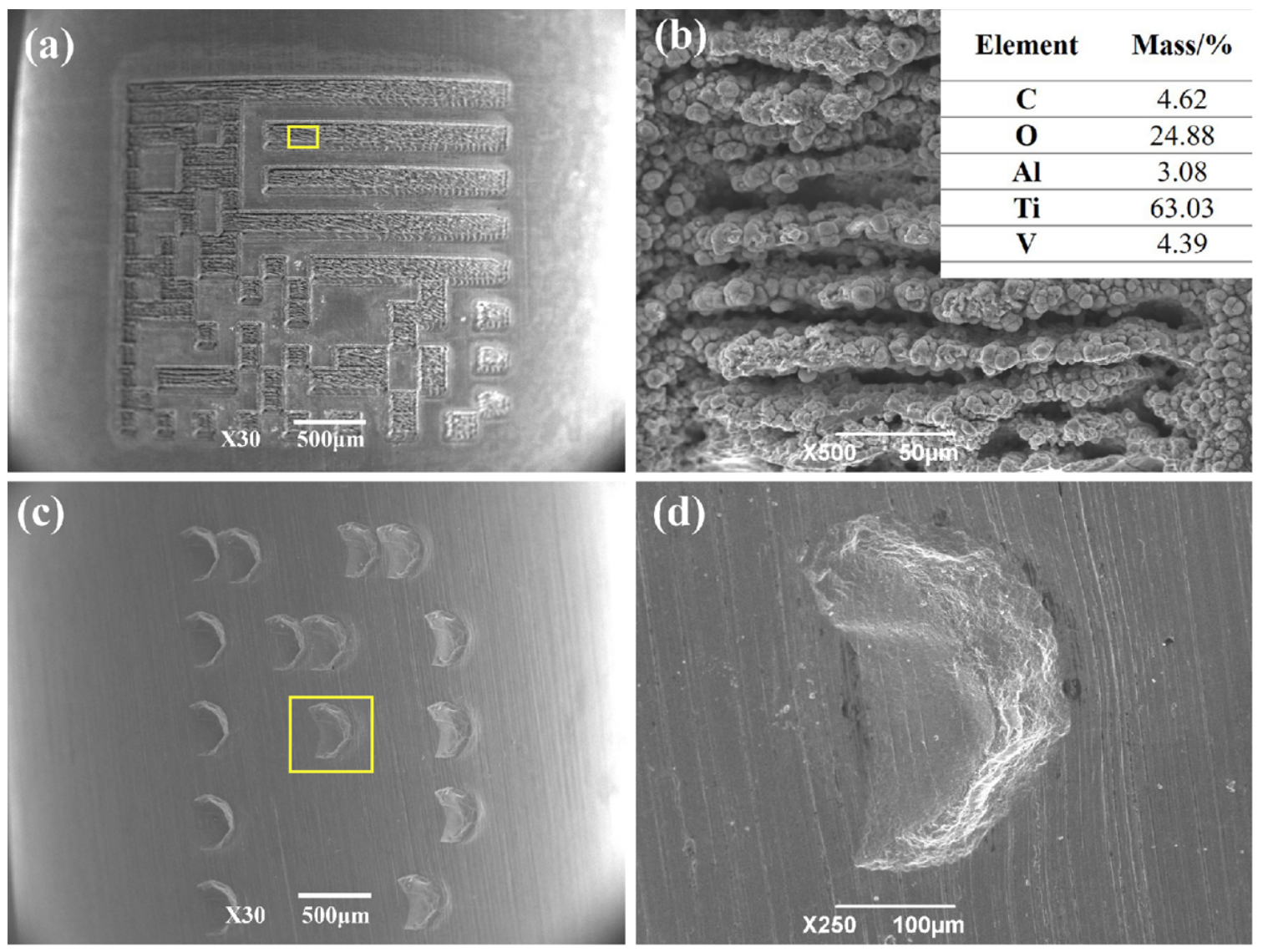

Fig. 1 (a) The morphology of surface damages induced by LM; (b) The magnified morphology of (a) and the element contents at the surface damages induced by LM; (c) The morphology of surface damages induced by MM; (d) The magnified morphology of (c).

\subsection{Fatigue performance}

Fig. 2 shows the fatigue life of BM, LM, USRP-LM, MM and USRP-MM treated samples at a maximum alternating stress of $700 \mathrm{MPa}$. Both of the marking methods were not conducive to the fatigue life of titanium alloy. The laser marking significantly decreased the fatigue life of titanium ally while the mechanical marking slightly decreased the fatigue life. This was related to the different surface damages caused by the DM codes. During the fatigue loading, the defects like micropores or cracks in the laser treated area were easy to become the fatigue crack initiation sites. As we know, the fatigue life is composed of fatigue crack initiation life and fatigue crack propagation life. The defects in the laser treated area greatly reduced the fatigue crack initiation life. Thus, the fatigue life of laser marked titanium alloy was reduced significantly. For the mechanical marked titanium alloy, though there were many pits on the titanium alloy surface, the fatigue life was slightly reduced which may be related to the local stress concentration at the bottom of the pits. Fig. 3 shows the fracture morphologies of BM, LM sample and MM sample. The cracks of all the samples were initiated on the titanium alloy surface. The BM showed a characteristic of single crack source (Fig. $3[a]$ and [b]). However, the crack initiation site of the laser marked sample showed a line source (Fig. 3[c] and [d]). This illustrated 
that the porous and loose layer had a detrimental effect on the fatigue life of titanium alloy. The fatigue cracks of mechanical marked sample were initiated at the bottom of the pits (Fig. 3[e] and [f]), which illustrated that the mechanical marking caused the stress concentration at the bottom of the pits.

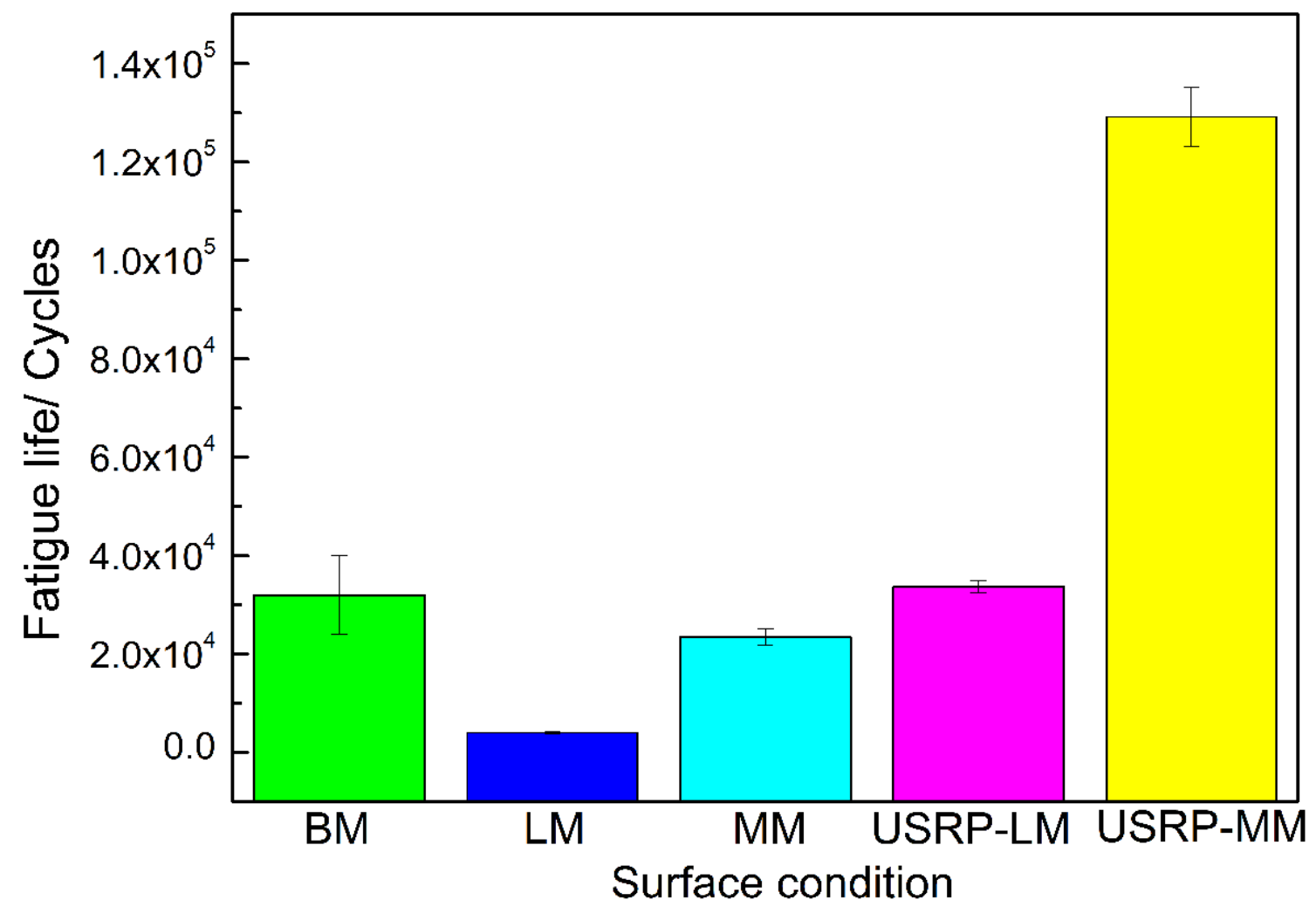

Fig. 2 The fatigue life of samples with different surface conditions at a maximum alternating stress of $700 \mathrm{MPa}$. 

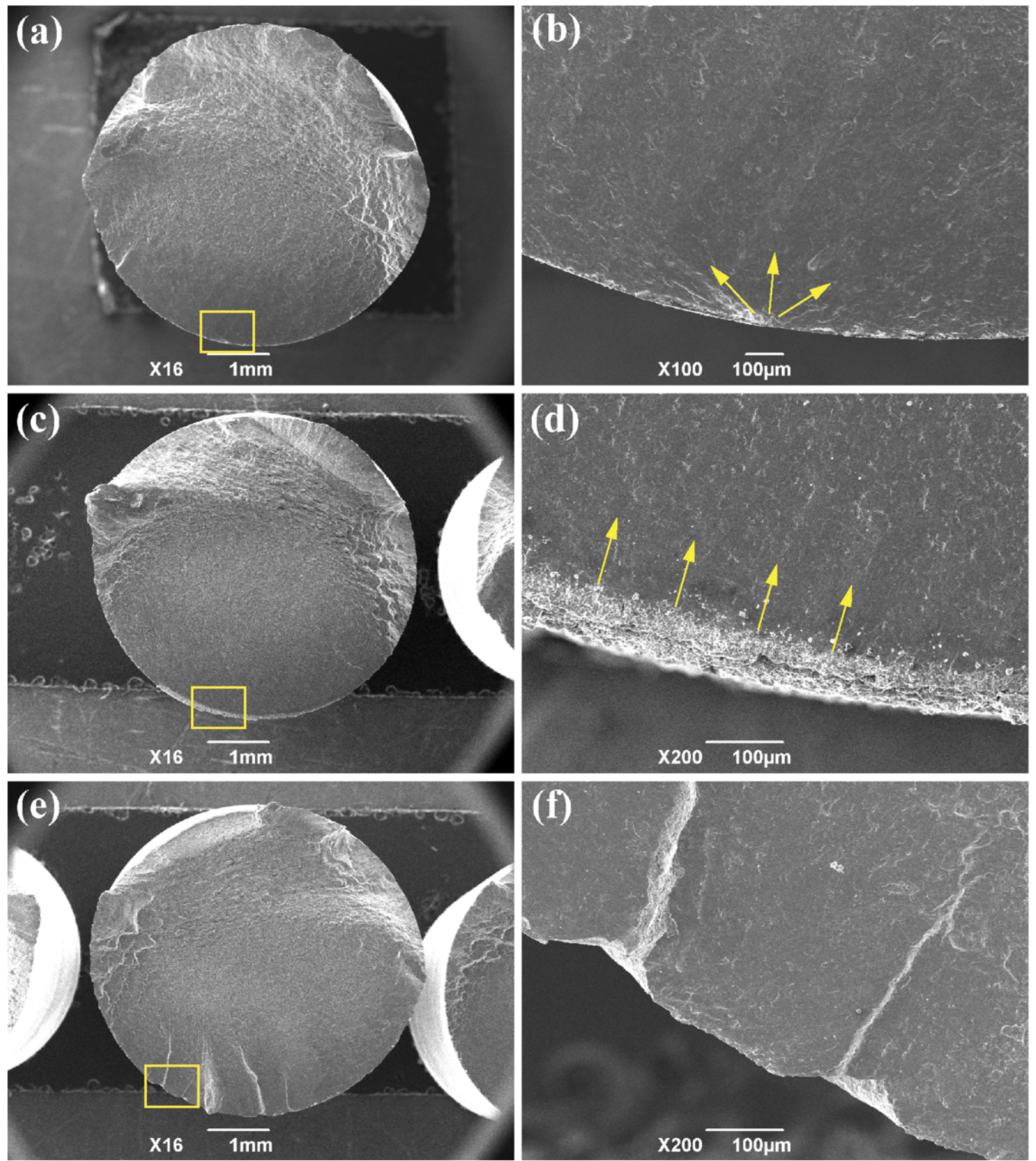

Fig. 3 Fracture morphologies of the samples with different surface conditions (a-b) the substrate; (c-d) LM; (e-f) MM.

The DM codes generally have a deleterious effect on the substrate due to surface damages caused by them. However, it is well known that surface mechanical treatments, like shot peening, surface mechanical rolling treatment and USRP, significantly improved the fatigue performance of metal materials $[6,10,11]$. Therefore, the sample was treated by USRP prior to marking to improve the fatigue performance of laser/mechanical marked titanium alloy. The results showed that USRP pretreatment did improve the fatigue performance of laser/mechanical marked titanium alloy. The fatigue life of LM sample was improved similar to that of the substrate via USRP pretreatment while the fatigue life of MM sample was improved by a factor of 3 via USRP pretreatment. This was mainly attributed to the compressive residual stress induced by USRP pretreatment. Our previous study [6] showed that USRP treatment could induce a large value compressive residual stress layer with a depth of $\sim 530 \mu \mathrm{m}$ at the titanium alloy surface layer. And the maximum value is $930 \mathrm{MPa}$ at the depth of $\sim 50 \mu \mathrm{m}$. It is well known that compressive residual stress could retard the fatigue crack initiation and fatigue crack early propagation [12]. However, the fracture morphologies of USRP-LM sample and USRP-MM sample showed that the fatigue crack initiation sites of the two samples were the same as that of LM treated sample and MM treated sample, which was initiated on the surface. This was different from the 
surface mechanical treated samples with no surface damage, the fatigue crack initiation sites of which were generally located at the material interior [6]. This illustrated that the compressive residual stress had little influence on the fatigue crack initiation sites but a big influence on the fatigue crack propagation. The compressive residual stress could not only decrease the tensile stress at the crack tip but also close up the cracks in the propagation early stage $[13,14]$. Thereby, the compressive residual stress slowed down the crack propagation rate.

Fig. 5 shows the side fracture morphologies of LM, USRP-LM, MM and USRP-MM samples. USRP pretreatment had little influence on the morphologies of the laser treated DM code or the mechanical treated DM code. The cracks of the laser marked samples were initiated at the complete oxidized side of the surface damage region (DM code region). Thus, it was suggested that the laser marked DM code should avoid the complete oxidized line of the code to improve the fatigue performance. In addition, the cracks of the mechanical marked sample were initiated at the pits. It was suggested that the process parameters of the mechanical marking should be improved to obtain rounder pits with less local stress concentration.
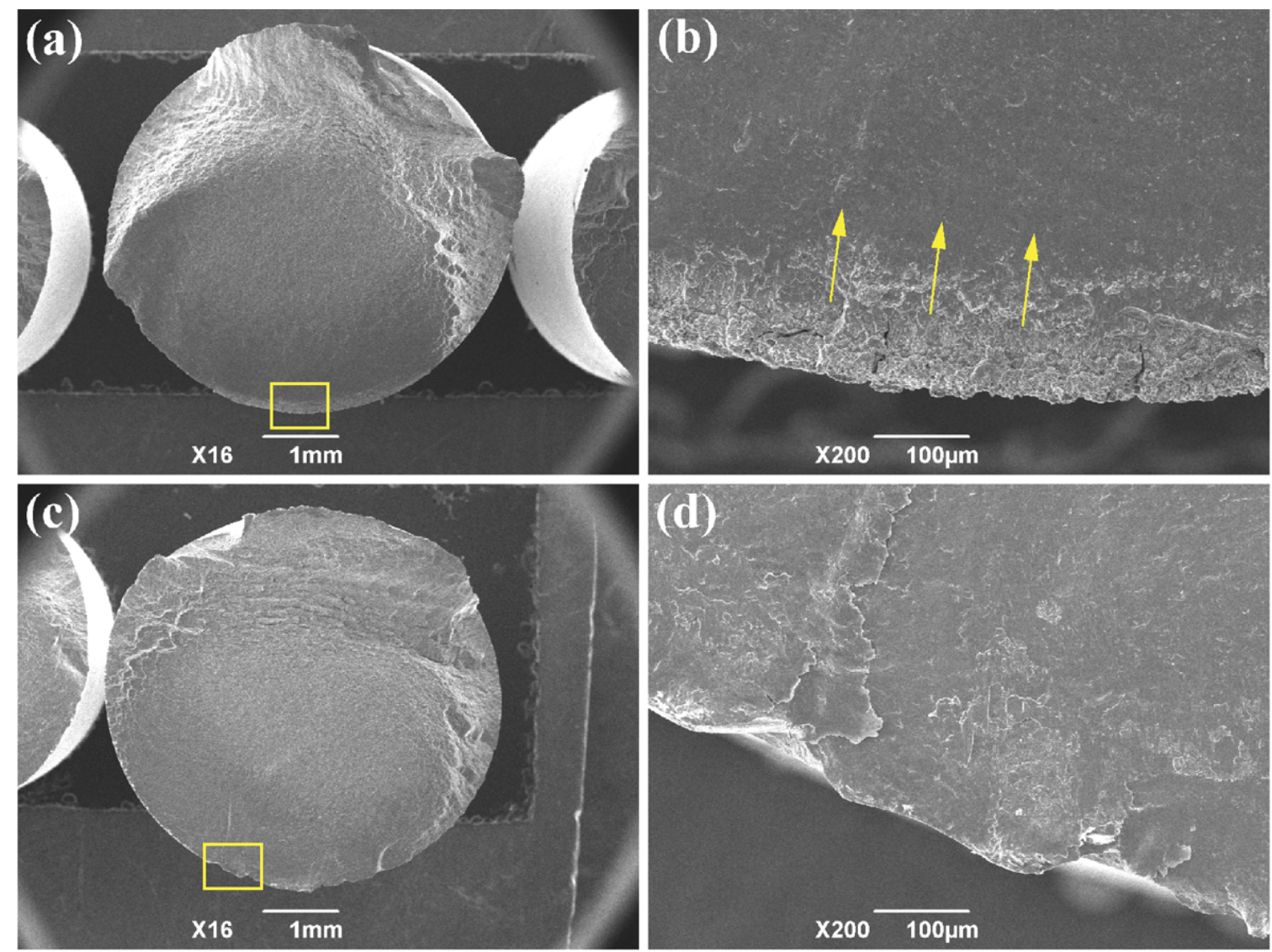

Fig. 4 Fracture morphologies of the samples with different surface conditions (a-b) USRP-LM; (c-d) USRP-MM. 

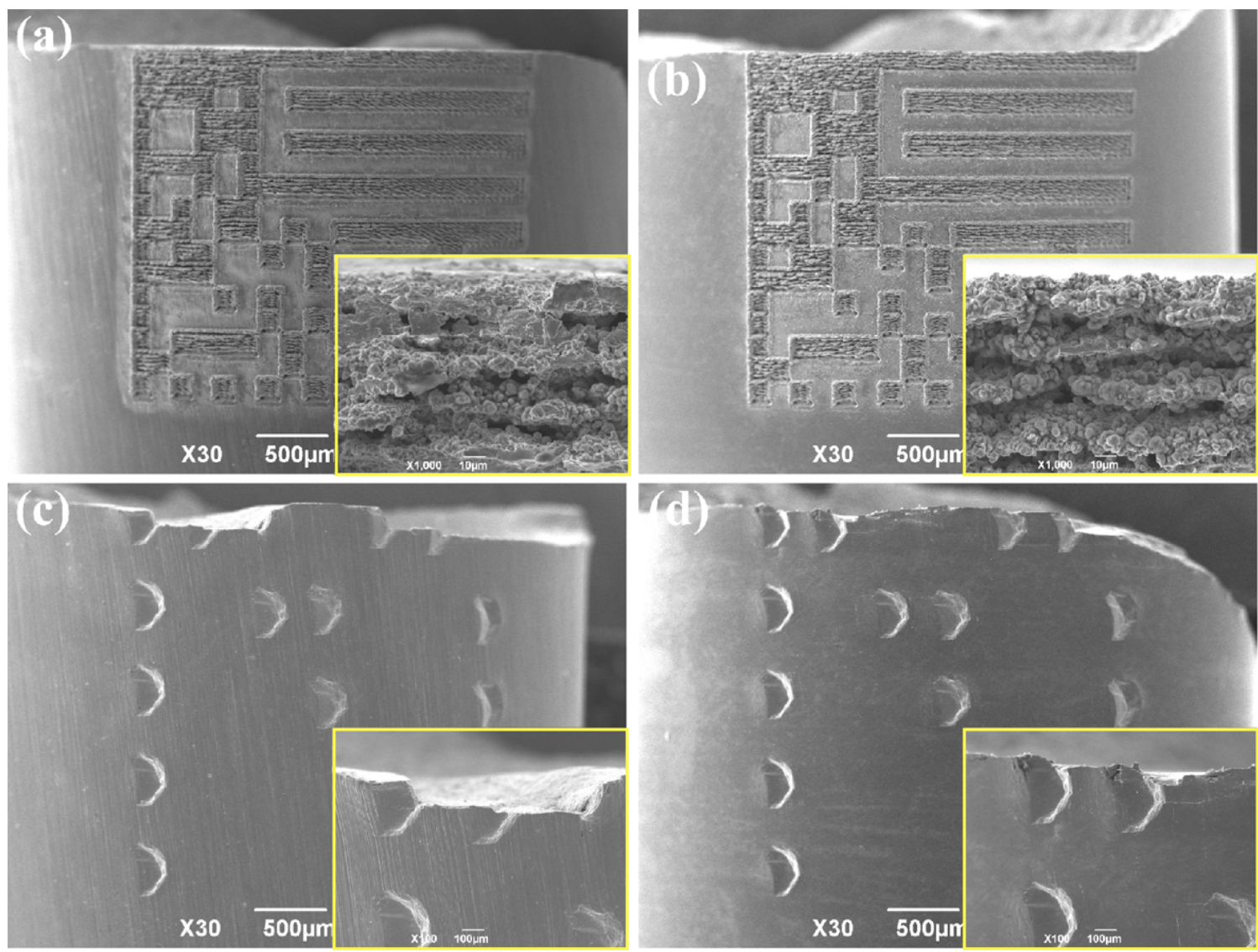

Fig. 5 Side fracture morphologies of the samples with different surface conditions (a) LM; (b) USRP-LM; (c) MM; (d) USRP-MM.

\section{$\underline{4 \text { Conclusion }}$}

(1) The laser marking caused a porous and loose oxidized layer with a depth of 40-60 $\mu \mathrm{m}$ on the titanium alloy surface while the mechanical marking caused many pits with a depth of 30-35 $\mu \mathrm{m}$ on the titanium alloy surface.

(2) Compared with the substrate, laser marking severely reduced the fatigue performance of the titanium alloy. This was attributed to the fact that laser marking produced a notch surface with loose oxide layer that was easy to initiate cracks. In addition, mechanical marking reduced the fatigue performance slightly for the bottom of the identification pits was blunt and therefore the local stress concentration caused by the pits was slight.

(3) USRP pretreatment significantly improved the fatigue life of mechanical marked sample while USRP pretreatment improved the fatigue life of laser marked sample similar to that of the substrate owing to that the large value and deepdistributed compressive residual stress retarded the fatigue crack early propagation.

\section{Acknowledgements}

This work was financially supported by the National Natural Science Foundation of China (51771155).

\section{$\underline{\text { References }}$}

[1] J. Qi, K.L. Wang, Y.M. Zhu, J Mater. Process Tech. 139(2003) 273-276. 
[2] C. Marx, M. Hustedt, H. Hoja, T. Winkelmann, T. Rath, Biosyst. Eng. 116(2013) 436-446.

[3] L. Costa, K. Lansford, D. Rajput, W. Hofmeister, Surf. Coat. Tech. 203(2009) 1984-1990.

[4] C. Velotti, A. Astarita, C. Leone, S. Genna, F. Minutolo, A. Squillace, Procedia CIRP 2016, pp. 975-980.

[5] G. Li, S. Qu, M. Xie, Z. Ren, X. Li, Materials 10(2017).

[6] C. Liu, D. Liu, X. Zhang, S. Yu, W. Zhao, Materials 10(2017).

[7] N. Ao, D. Liu, X. Xu, X. Zhang, D. Liu, Mater. Sci. Eng. A. 742(2019) 820-834.

[8] A. Leoni, I. Apachitei, A.C. Riemslag, L.E. Fratila-Apachitei, J. Duszczyk, Mater. Sci. Eng. C. 31(2011) 1779-1783.

[9] F. Vargas, H. Ageorges, P. Fournier, P. Fauchais, M.E. Lopez, Surf. Coat. Tech. 205(2010) 1132-1136.

[10] S. Kumar, K. Chattopadhyay, V. Singh, J Alloy Compd. 724(2017) 187-197.

[11] H.W. Huang, Z.B. Wang, J. Lu, K. Lu, Acta Mater. 87(2015) 150-160.

[12] S. Kumar, K. Chattopadhyay, V. Singh, J Alloy Compd. 724(2017) 187-197.

[13] D.X. Liu, B. Tang, X.D. Zhu, H. Chen, J.W. He, J.P. Celis, Surf. Coat. Tech. 116(1999) 234-238.

[14] R.B. Waterhouse, A.J. Trowsdale, J Phys. D Appl. Phys. 25(1992) A236-A239. 